\title{
Morphological Change Detection System for Real Time Traffic Analysis
}

\author{
Anuradha S. G. ${ }^{1}$, K. Karibasappa ${ }^{2}$ and B. Eswar Reddy ${ }^{3}$ \\ ${ }^{1}$ Research Scholar Department of CS\&E, JNTUA College of Engineering, Anantapur \\ 515002 \\ ${ }^{2}$ Department of IS\&E, Dayananda Sagar College of Engineering, Bangalore, India \\ ${ }^{3}$ Department of CS\&E, JNTUA College of Engineering, Anantapur 515002, India \\ Iemail-id:anuradha_gadagin@rediffmail.com \\ ${ }^{2}$ email-id:karibasappa@hotmail.com \\ 3email-id:eswarcsejntu@gmail.com
}

\begin{abstract}
Nowadays roads are getting overcrowded and the number of vehicles on the roads is increasing. The main reason is the increase in the population of metro cities, urbanization, and economical development of the country that subsequently led to the increased demand for vehicular travel. Incident detection and Traffic congestion are the serious issues in Traffic engineering applications and Intelligent Transport System. Hence the aim of the proposed system is to build an automatic traffic monitoring system which can replace or reduce manual traffic monitoring. The proposed mathematical Morphogical technique is able to detect and track the moving vehicle from the traffic video in real time and give a message to the traffic control station. The experimental results show that the proposed technique can be adopted under various traffic patterns, weather conditions and illuminations.
\end{abstract}

Keywords: Edge detection, Background Subtraction, Image Processing, Segmentation, Object Tracking, Traffic Analysis, Morphogical operations

\section{Introduction}

The application of Morphogical Image Processing and Computer vision techniques to the real time traffic analysis provides an efficient automatic system for traffic data collection and monitoring when compared with the traditional methods. Manual Traffic Monitoring systems involving direct observations of human observers suffer from serious difficulties due to weather conditions, staffing limitations etc. Traffic patterns can be acquired using sensor technology, inductive loops detectors, sonar detectors and microwave detectors can also be used for detection and counting but their installation and maintenance makes the traffic disruptions. Apart from these sensors used in the traffic monitoring systems can be damaged by snow, fog, change in temperature and humidity making the detection task more badly. These limitations in the critical situation have motivated the researches to an active field of research in detecting moving vehicle in static and dynamic environment by capturing live video of the scene [10]. A number of techniques and algorithms have been proposed in the past for detecting the moving vehicle in the video sequence [1-6]. Each of these have their particular features and helps in the analysis of traffic flows, turning movement of vehicles, speed enforcement, vehicle count, vehicle classification, traffic congestion, highway state assement, autonomous vehicle guidance and accident investigation. Over the past few years, the volume of interest, research and development of Morphological image processing techniques for change detection in vision based and surveillance systems has increased tremendously in contrast with the traditional threshold and fuzzy logic techniques [5-7]. 


\section{Related Work}

In a survey paper, Saket Bhardwaj and Ajay Mittal [1] presented a comparison between various edge detectors and concluded that Modified declivity operator gives better result when compared to prewitt, sobel, canny, Roberts and LoG edge detectors. Modified declivity operator does not require preprocessing and it's a non linear differential operator [1].Edges play a very important role in object recognition. Edge characterizes boundaries of objects in an image and should always precede the closed contour. Edge detection finds its practical application in the medical diagnostics, segmentation, and computer guided surgery, fault detection, website tracking, monitoring, satellite imaging, face recognition, spam filtering, finger print recognition, automated traffic controlling system and many more to name in the field of recognition, tracking and visual surveillance systems $[12,14]$. The work on the revising the canny edge detector focused to accurately detect and classify the edge as either true edge or false edge [2].The method described showed the accurate results of classifying the edge elements and accurately recover all the edges missed by canny edge detector [2]. A single edge pixel if it is missing then significant information associated with that pixel is lost and there after the closed contour will turn to open. Such a classification will disable us from detecting the object of interest and in future have its impacts on recognition and tracking applications. Pinaki Pratin Achariya,Ritaban Das \& Dibyendu Ghoshal presented a comparative analysis of various edge detectors [4].It is observed from their study that canny edge detector performed well when compared with gradient operators with a higher Entropy, Peak Signal to Noise Ratio(PSNR),Mean Square Error(MSE).The execution time are studied and analyzed w.r.t to various edge detectors [4]. A Soft Computing technique based on Fuzzy set is proposed by Pushpajit A.Kharie and Dr.Nileshsingh V.Thakur [5] for edge detection. The image here is considered as a fuzzy set and the pixels are taken as the elements of fuzzy set. The fuzzy approach by making use of two threshold levels i.e. higher threshold and lower threshold computes both the weak edge and the strong edge [5]. Elena Stringa [6] proposes Morphogical Change Detection algorithms for Visual Surveillance and remote monitoring applications. The algorithm proposed in their work is based on novel Morphogical image processing in contrast with the regular change detection algorithms using MIP. The method is designed to real time systems of computer vision systems and does not require any system parameter settings [6].

In contrast to the earlier Threshold Edge Detection methods, Proposed a fuzzy logic approach [7]. Fuzzy image processing is a prominent tool for accurately deciding the edge and tries as much as possible to improve the quality of true edge. Apart from the system being robust to varying condition over time, it requires extensive computation. In a survey paper, N.SenthiKumaran and R.Rajesh [8] presented the theory of edge detection for Segmentation. The study focused mainly on partitioning the images into meaningful regions and extracting the significant information which can later be used for high level image processing applications using Genetic algorithms, Fuzzy Logic techniques and Neural Computing techniques [8]. The investigation of Canny Edge Detection algorithm and Gabor Method were carried to identify the points at which the image intensity changes sharply or slowly or remains same in the digital images [9] .The results of the work highlight on the number of adjustable parameters like the size of Gaussian filter and use of two thresholds (low, high) which effect on efficiency of the algorithm and also the computation time [9]. Traffic Monitoring System using run average technique is proposed to detect and track moving objects [10]. The adaptive method is developed by considering both static and dynamic backgrounds in video sequences and different camera parameters. The system can deal with slows light changes to detect, track and remove shadow effect and reduces the task of human monitoring in various applications like a road in metro cities, cricket match to track players, cricket ball, empires etc, and people counting in the public meetings etc. [10]. A fast, robust, automatic segmentation algorithm is proposed in [11] to extract the semantically meaningful 
objects in the image sequences. The authors in their work described a comparative study on various change detection algorithms and model matching techniques.

Many of changeling real time issues in the field of video image processing such as illumination changes, rain, snow and shadows in moving background are addressed by SenChing S. Cheung and Chandrika Kamath [12]. Various background subtraction methods are studied, analyzed and tested with urban traffic videos by using frame differing, adaptive median filtering, median filtering, kalman filtering and mixture of Gaussians. The study observed the best results with mixture of Gaussians model and adaptive median filtering and directs the researchers to a new problem on to improve balance between fast adaption and robust system [12]. Edge Detection is an essential tool in computer vision as because it gives the clear separation between the background and the objects in an image [1,2]. If the edges of the image are clearly identified, all the objects can be located and further classified which aids in recognition and classification of objects in an image sequences. A comparative study and evaluation report using various edge detectors with number of measuring metrics is presented in [13]. A vision based system for road traffic monitoring is the vital application in the computer vision. A survey paper [14] focuses on the various state of the art algorithms and the tools used in traffic applications. Automatic lane finding and vehicle detection appears to be trivial but are not so simple in real time $[14,12]$ and hence are the challenging and active areas research video image processing.

\section{Theoretical Background}

Edge Detection and Change Detection are the prominent steps in segmentation, detection, tracking and recognition of the object in the scène of interest. Edge Detection is a low level operation which reduces the amount of data by removing the unnecessary information and providing only the necessary information i.e. detecting the object of our interest.

\subsection{Edge Detection}

The sharp points or changes in the threshold of a digital image results to an edge in digital image. The detection of edge in a digital image is very important and challenging task in case of noisy images, image subjected to various illuminations, reflections, shadow casts of images etc. In all such deciding whether pixel is edge or not becomes difficult and false edges are thus identified. Thus in real time applications eliminating the false edges and reducing the misclassification error is very important. Edge detection plays a prominent step in segmentation, object detection, tracking and recognition applications. Different edge detection techniques that are considered in our work include:

- Sobel operator

- Roberts operator

- Prewitt operator

- Canny edge detector

- Morphogical edge detector

- Background subtraction

\subsection{Change Detection}

Change detection is the process to identify the times when the change occurs in time series. It is a probabilistic approach which not only measures the intensity changes over the time but also detects whether or not a change has occurred or several changes have occurred and also identifies the time at which such changes occur. Practical motivation of change detection primarily focuses on Video Segmentation and further on Video Object Recognition and Tracking. Change detection methodology provides a preferential tool for automatic segmentation which can either be offline or online. Our paper mainly focuses on building an automatic system in real time to detect the vehicle, track it and measure speed of vehicles 
from the real time traffic video sequence. The system can further aid in monitoring and controlling the traffic in metro cities, estimating the crowd in cricket stadium and other video surveillance applications. Canny edge detector is the best among the other edge detectors to detect the changes in the images subjected to noise, local changes in the threshold, illumination changes etc. The classical edge detection techniques mentioned above still suffer from object blur and can not accurately handle the changes in weather conditions and illumination. To deal with these problems before performing canny edge detection, a binary morphology a pre-processing step is carried out and later is accompanied with Morphogical post processing operations in our proposed work.

\section{Implementation}

Numerous work in the field of video analysis specially in the area of video object detection, video object segmentation, co-object segmentation and tracking are proposed in $[6,12,14-16]$. The proposed Morphogical change detection algorithm is implemented using Matlab and is the experimental work based on my previous research work already published [15-16].The aim of the current work is to separate the object of interest i.e. vehicle, and further count the number of vehicles and display the alert messages to the traffic control system. Motion cue present in the image frame is used for change detection. And based on the temporal motion information present in $\mathrm{i}^{\text {th }}$ and (i-1) image frame, block comparison is carried out. For the first time the current frame is compared against frame extracted when no vehicles are on the road. In the first step traffic videos of highways are captured by means of the camera and are fed as the input to the system. Secondly preprocessing is done on the videos to eliminate noise, illumination and other reduce other effects resulted from the camera. Thus the result obtained is the high quality video and is latterly converted to grayscale .The grayscale video is subjected thirdly to frame extraction step. The next step in the framework does motion detection using the traditional background subtraction technique to detect the moving objects followed by a pre-processing step binary morphology. The final step to track the vehicle is to detect the change in the image sequence accomplished with Morphogical post processing operations. The detail steps used in the implementation of Morphological Change Detection System for Real Time Traffic Analysis are shown below in Figure1.

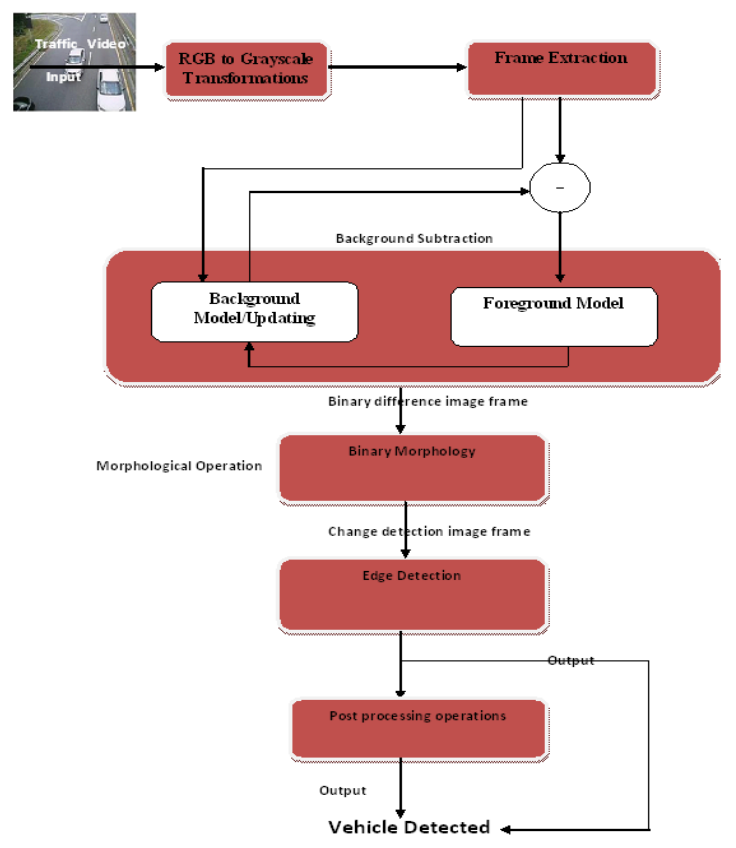

Figure 1. Morphogical Change Detection Framework 


\section{Experimental Tests and Results}

The experimental work is carried out to MATLAB using CV datasets on the web.html and SVCL datasets. The videos with different traffic conditions, illumination conditions and weather conditions [sunny day, rainy day, normal day light and night light conditions, no traffic, less traffic, heavy traffic] are variously addressed. Firstly we perform some experiments on the traffic videos in real time using proposed mathematical morphological operators to detect the change and compare it with other edge detectors. The result thus obtained can be used in real traffic monitoring and controlling systems.

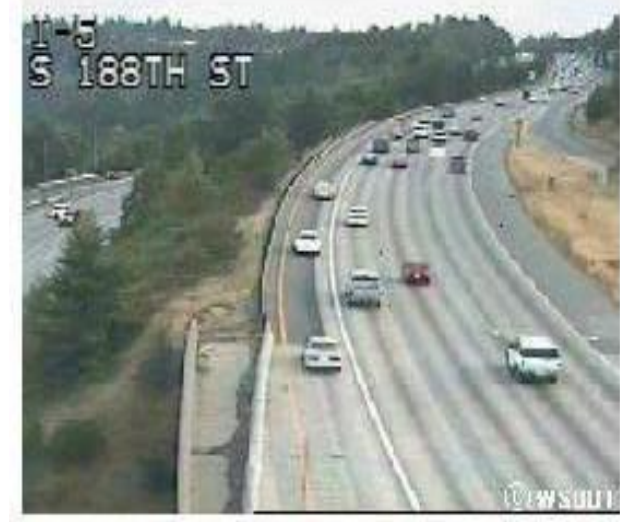

(a)Original image

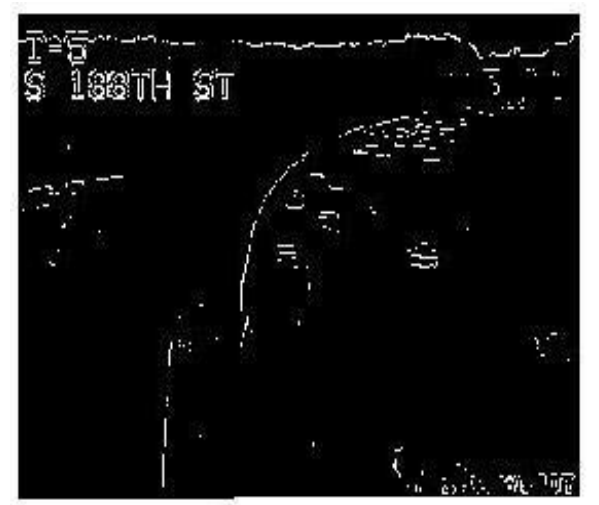

(c) Roberts Cross Edge Detector

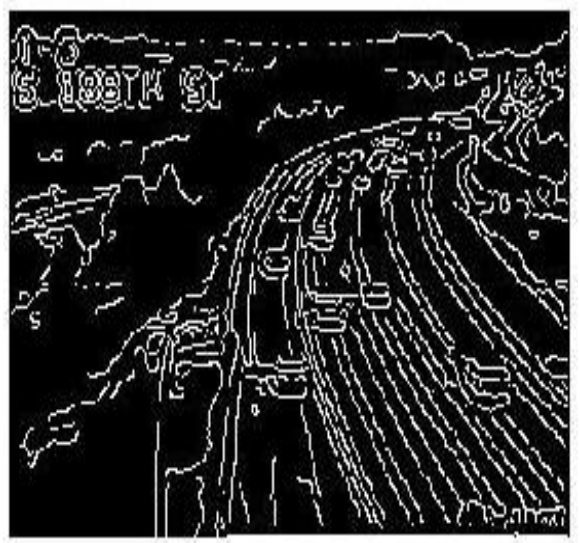

(e) Canny Edge Detector

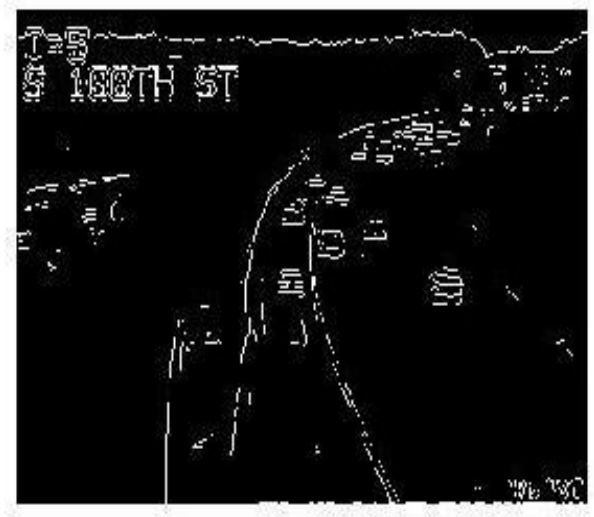

(b) Sobel Edge Detector

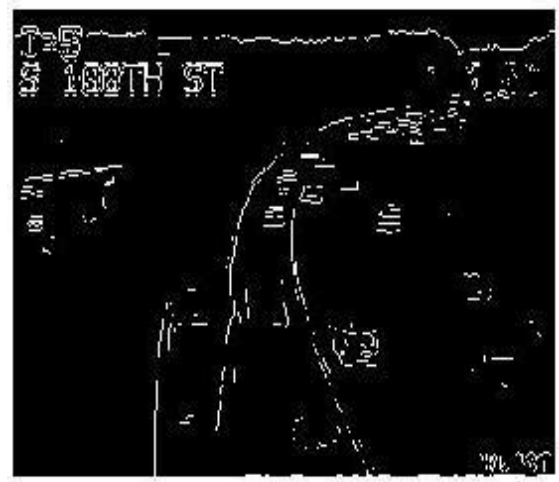

(d) Prewitt Edge Detector

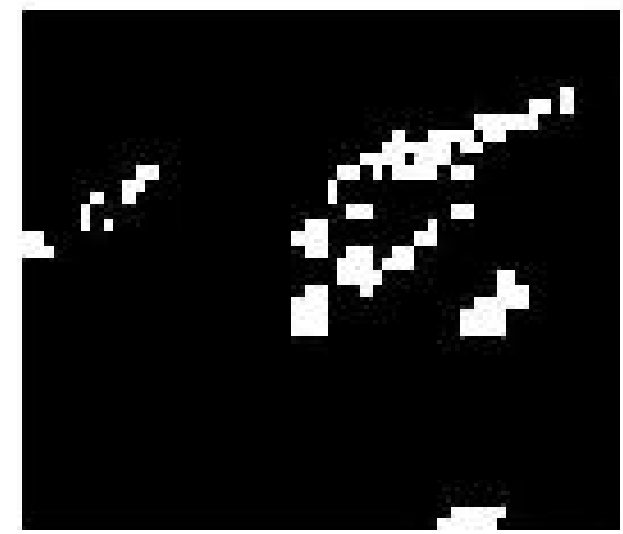

(f) Background Subtraction 


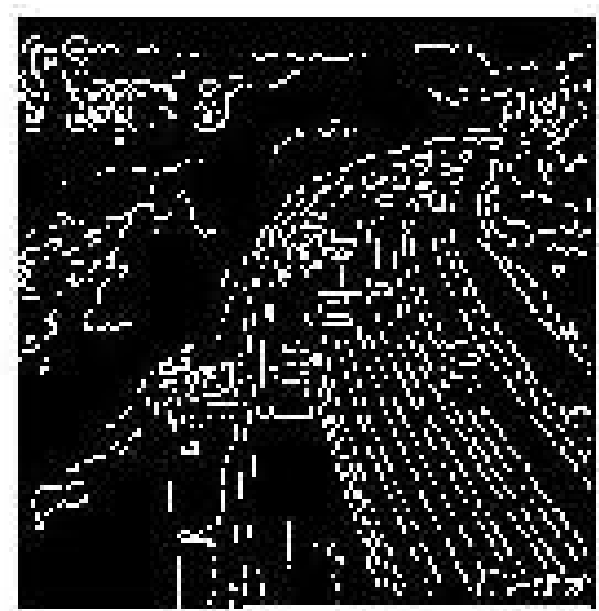

(g) Proposed Morphological Edge Detector

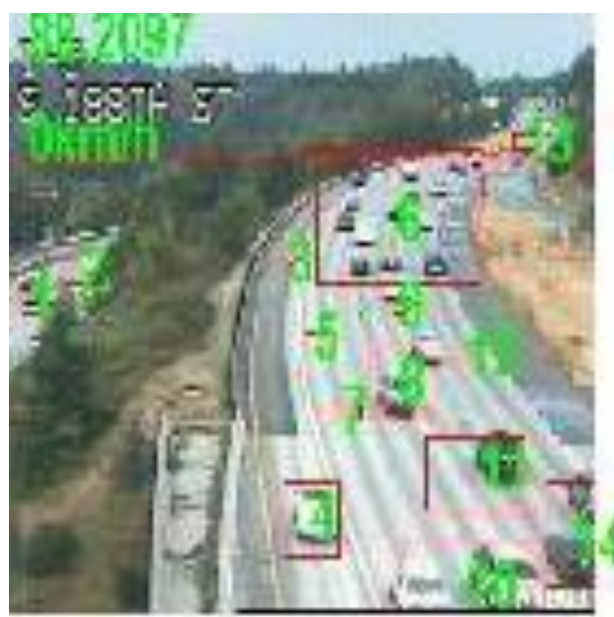

(h) Vehicle Tracking

Secondly according to the queuing theory, inter-arrival time and inter-departure time are the two performance metrics being evaluated for video traffic analysis and monitoring system in our work and the respective results are tabulated in table1 as shown in .The interarrival time and inter-depature time is tabulated in the below table1 by appliying various edge detectors to the taffic video sample.From the table 1, we can easily give the compartive result on the various edge detectors that canny edge detector is the optimal one for video traffic analysis giving a better inter-arrival rate. Finally the real time traffic analysis and monitoring system performs the analysis on the traffic patterns based on the size of the traffic and a message is shown in a window. If the traffic condition is less then message shown is "Normal" and if the traffic is heavy then message shown is "Traffic" as in the below figure 4(a) and 4(b).

\section{Table 1. Inter-arrival Time and Inter-depature Time of Sample Video w.r.t Various Edge Detector}

\begin{tabular}{|l|l|l|}
\hline \multicolumn{1}{|c|}{ Edge Detectors } & \multicolumn{1}{c|}{ Inter-arrival Time (IAT) } & Inter-departure Time (IDT) \\
\hline Canny Edge Detector & 10.1115 & 2.514 \\
\hline Sobel Operator & 9.2030 & 2.234 \\
\hline Roberts Cross Operator & 8.6038 & 1.955 \\
\hline Prewitt Operator & 8.6806 & 1.396 \\
\hline
\end{tabular}

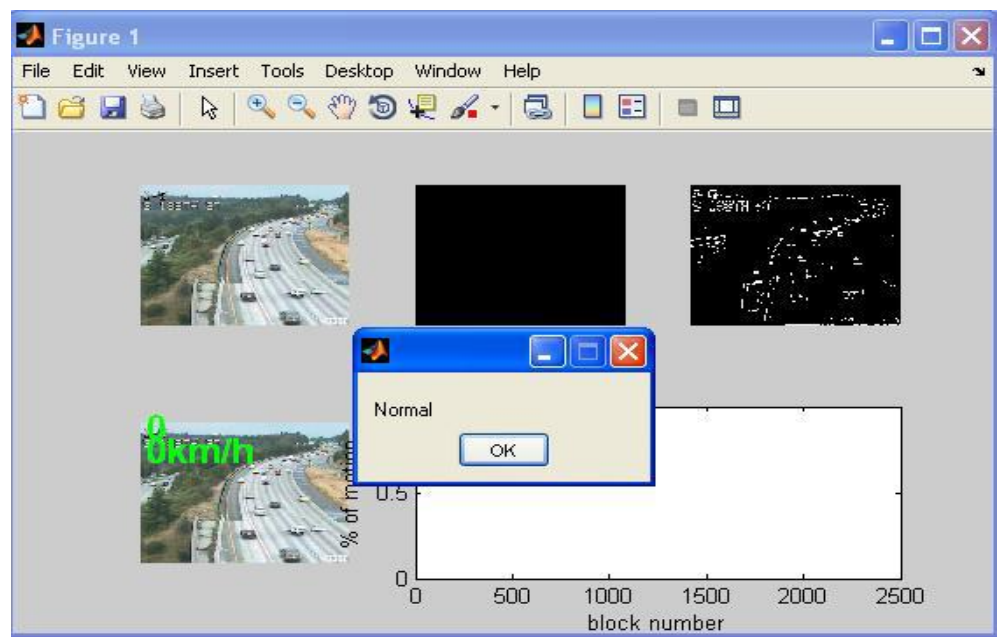

Figure 4(a). Normal Traffic Condition 


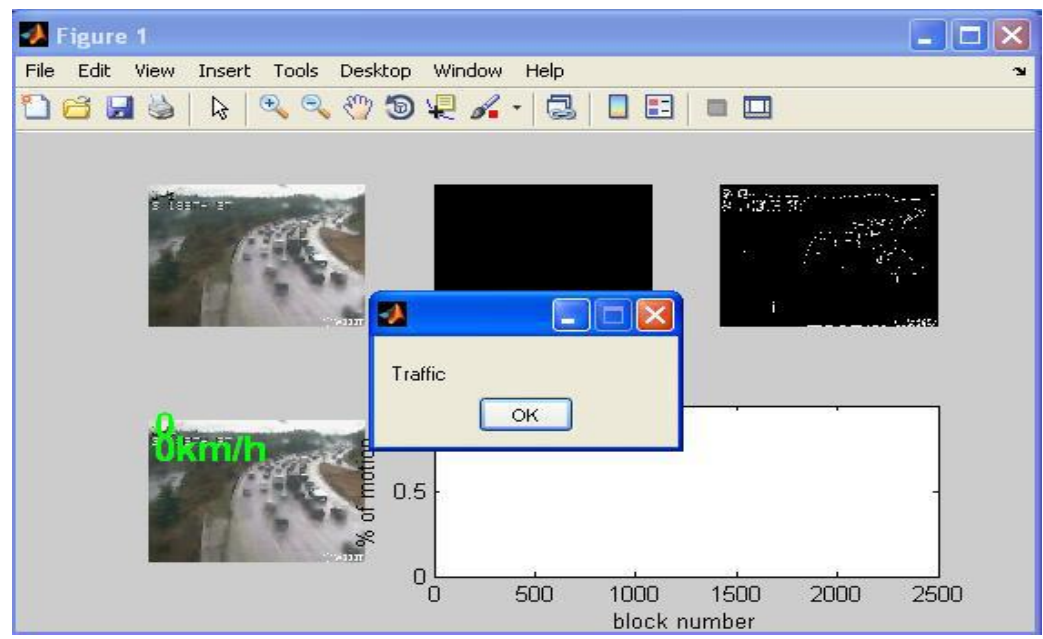

Figure 4(b). Heavy Traffic Condition

\section{Conclusion}

The paper presents a robust, semi-automated real-time traffic monitoring and controlling system. Morphogical image processing approach is proposed in the work for detecting the change in the traffic patterns, estimating the traffic and also in counting the number of vehicles in the observed video. The input to the semi automated system is the traffic video of the highway and the analysis is done on each frame of the image sequence separately by setting the threshold. The output of the system is alert message extracted from the attributes of the traffic patterns. Two messages are displayed to control the traffic i.e. "Traffic" and "Normal" based on the total number of vehicles more than the set threshold or less than the set threshold respectively. This intelligent traffic control system reduces or replaces the job of human traffic controller and is also an advantage over the other automated systems because of its accuracy .It offers a cheaper solution in traffic analysis as senor are not used as in the past. The experimental work is also carried out by using the proposed Morphogical edge detectors and is compared with Sobel operator, Roberts's operator, Prewitt operator, canny edge detector and the traditional background subtraction method. The proposed algorithm is efficient and yields good result as it is suitable to monitor the traffic under different weather conditions and illumination changes.

\section{References}

[1] S. Bhardwaj and A. Mittal, "A Survey on Various Edge Detector Techniques", 2nd International Conference on Computer, Communication, Control and Information Technology (C3IT-2012) on February 25 - 26, 2012, Procedia Technology, vol. 4, (2012), pp. 220-226.

[2] L. Ding and A. Goshtasby, "On the Canny edge detector", The Journal of Pattern Recognition Society PERGAMON, vol. 34, (2001) pp. 721-725.

[3] M. Abo-Zahhad, R. Gharieb, S. Ahmed and A. Donkol, "Edge Detection with a Preprocessing Approach", Journal of Signal and Information Processing, vol. 5, (2014), pp. 123-134.

[4] P. P. Acharjya, R. Das and D. Ghoshal, "Study and Comparison of Different Edge Detectors for Image Segmentation”, Global Journal of Computer Science and Technology Graphics \& Vision, vol. 12, no. 13, Version 1.0, (2012).

[5] P. A. Khaire and N. V. Thakur, "A Fuzzy Set Approach for Edge Detection”, International Journal of Image Processing (IJIP), vol. 6, (2012), pp. 403-412.

[6] E. Stringa, "Morphological Change Detection Algorithms for Surveillance Applications", British Machine Vision Association, (2000), In BMVC, pp. 1-10.

[7] A. A. Alshennawy and A. A. Aly, "Edge detection in digital images using fuzzy logic technique", World Academy of science, engineering and technology, vol. 51, (2009), pp. 178-186.

[8] P. A. Hajare and P. A. Tijare, "Edge Detection Techniques for Image Segmentation", International Journal of Computer Science And Applications, vol. 4, no. 1, (2011). 
[9] M. Nosrati, R. Karimi, M. Hariri, and K. Malekia, "Edge Detection Techniques in Processing Digital Images: Investigation of Canny Algorithm and Gabor Method", World Applied Programming, vol. 3, no. 3, (2013), pp. 116-121.

[10] J. Zhang and C. H. Chen, "Moving Objects Detection and Segmentation In Dynamic Video Backgrounds "Technologies for Homeland Security, 2007 IEEE Conference on 16-17 May 2007, pp. 64 - 69, E-ISBN: 14244-1053-5, Print ISBN:1-4244-1053-5.

[11] K. Ganesan and S. Jalla, "Video Object Extraction Based On A Comparative Study of Efficient Edge Detection Techniques", the International Arab Journal of Information Technology, vol. 6, no. 2, (2009) April.

[12] S.-C. S. Cheung and C. Kamath "Robust techniques for background subtraction in urban traffic video", Visual Communications and Image Processing, 2004, vol. 5308, no. 1, (2004), pp. 881-892.

[13] E. Nadernejad, S. Sharifzadeh and H. Hassanpour, "Edge detection techniques: evaluations and comparison", Applied Mathematical Sciences, vol. 2, no. 31, (2008), pp. 1507-1520.

[14] V. Kastrinaki, M. Zervakis and K. Kalaitzakis, "A survey of video processing techniques for traffic applications", Image and vision computing, vol. 21, no. 4, (2003), pp. 359-381.

[15] Y. M. Prutha and S. G. Anuradha, "Morphological Image Processing Approach of Vehicle Detection for Real-Time Traffic Analysis", International Journal of Engineering Research \& Technology, vol. 3, no. 5, (May - 2014), e-ISSN: 2278-0181.

[16] Y. M. Prutha and S. G. Anuradha, "Morphological Image Processing Approach of Vehicle Detection for Real-Time Traffic Analysis”, International Journal of Computer Sciences and Engineering, vol. 2, no. 5, Page No (80-92), May -2014, E-ISSN: 2347-2693. 